\title{
Texture Analysis to Detect Cerebral Degeneration in Amyotrophic Lateral Sclerosis
}

\author{
Abdullah Ishaque, Rouzbeh Maani, Jerome Satkunam, Peter Seres, Dennell Mah, \\ Alan H. Wilman, Sandeep Naik, Yee-Hong Yang, Sanjay Kalra
}

\begin{abstract}
Background: Evidence of cerebral degeneration is not apparent on routine brain MRI in amyotrophic lateral sclerosis (ALS). Texture analysis can detect change in images based on the statistical properties of voxel intensities. Our objective was to test the utility of texture analysis in detecting cerebral degeneration in ALS. A secondary objective was to determine whether the performance of texture analysis is dependent on image resolution. Methods: High-resolution $\left(0.5 \times 0.5 \mathrm{~mm}^{2}\right.$ in-plane $)$ coronal T2-weighted MRI of the brain were acquired from 12 patients with ALS and 19 healthy controls on a 4.7 Tesla MRI system. Image data sets at lower resolutions were created by down-sampling to $1 \times 1,2 \times 2,3 \times 3$, and $4 \times 4 \mathrm{~mm}^{2}$. Texture features were extracted from a slice encompassing the corticospinal tract at the different resolutions and tested for their discriminatory power and correlations with clinical measures. Subjects were also classified by visual assessment by expert reviewers. Results: Texture features were different between ALS patients and healthy controls at $1 \times 1,2 \times 2$, and $3 \times 3 \mathrm{~mm}^{2}$ resolutions. Texture features correlated with measures of upper motor neuron function and disability. Optimal classification performance was achieved when best-performing texture features were combined with visual assessment at $2 \times 2 \mathrm{~mm}^{2}$ resolution ( 0.851 area under the curve, $83 \%$ sensitivity, $79 \%$ specificity). Conclusions: Texture analysis can detect subtle abnormalities in MRI of ALS patients. The clinical yield of the method is dependent on image resolution. Texture analysis holds promise as a potential source of neuroimaging biomarkers in ALS.
\end{abstract}

RÉSUMÉ: Utiliser l'analyse texturale pour détecter la dégénérescence cérébrale induite par la sclérose latérale amyotrophique. Contexte : Des indices de dégénérescence cérébrale ne sont pas manifestes lorsqu'on effectue un test d'imagerie par résonance magnétique (IRM) dans le cas de patients atteints de sclérose latérale amyotrophique (SLA). En se fondant sur les propriétés statistiques de l'intensité des voxels, l'analyse texturale peut permettre de détecter des altérations dans les images ainsi obtenues. Notre principal objectif a donc été de tester l'utilité de l'analyse texturale dans le dépistage d'indices de dégénérescence cérébrale chez des patients atteints de SLA. Un autre objectif, secondaire celui-là, a été de déterminer si l'efficacité de l'analyse structurale était tributaire de la résolution des images. Méthodes : Au moyen d'un système d'IRM Tesla 4.7, des images par coupes coronales du cerveau à haute résolution (résolution spatiale de $0,5 \times 0,5 \mathrm{~mm}^{2}$ ) en pondération T2 ont été obtenues chez 12 patients atteints de SLA et chez 19 individus en santé d'un groupe témoin. Des ensembles de données d'imagerie à des résolutions inférieures ont également été générés à partir d'une technique de sous-échantillonnage à $1 \mathrm{x}$ $1,2 \times 2,3 \times 3$ et $4 \times 4 \mathrm{~mm}^{2}$. À différentes résolutions, on a ainsi pu extraire des caractéristiques texturales à partir d'une tranche englobant le faisceau corticospinal et procédé à des tests évaluant leur capacité discriminante et leur valeur corrélative au moyen d'indicateurs cliniques. Des examinateurs experts ont également classé les sujets à l'étude à la suite d'une évaluation visuelle. Résultats : Les caractéristiques texturales des patients atteints de SLA se sont révélées différentes de celles des individus du groupe témoin pour les résolutions suivantes : 1 x 1, 2 × 2 et $3 \times 3 \mathrm{~mm}^{2}$. Nous avons aussi pu établir une corrélation entre les caractéristiques texturales et des indicateurs de fonctionnement et d'incapacité du motoneurone supérieur. Un classement optimal des résultats a été obtenu lorsque les caractéristiques texturales les plus efficaces ont été combinées à une évaluation visuelle utilisant une résolution de 2 x $2 \mathrm{~mm}^{2}$ (aire sous la courbe : 0,851; sensibilité de $83 \%$; spécificité de $79 \%$ ). Conclusions : L'analyse texturale permet de détecter des anomalies subtiles dans le cas d'IRM obtenues chez des patients atteints de SLA. L'efficacité clinique de cette méthode est aussi tributaire de la résolution des images. Dans le cas de la SLA, on peut donc soutenir que l'analyse texturale augure d'un avenir prometteur à titre de source potentielle de biomarqueurs dérivés de la neuro-imagerie.

Keywords: Amyotrophic lateral sclerosis, MRI, Diagnostic neuroradiology

doi: $10.1017 / \operatorname{cjn} .2018 .267$

Can J Neurol Sci. 2018; 45: 533-539

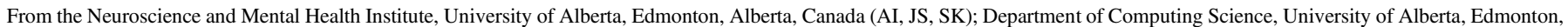

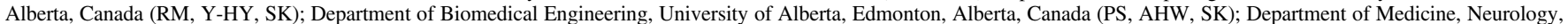

University of Alberta, Edmonton, Alberta, Canada (DM, SK); Department of Radiology and Diagnostic Imaging, University of Alberta, Edmonton, Alberta, Canada (SN).

Received December 27, 2017. Final Revisions Submitted May 3, 2018. Date of Acceptance May 10, 2018.

Correspondence to: S. Kalra, 7-132F Clinical Sciences Building, 1135083 Ave. NW, Edmonton, AB, Canada T6G 2G3. Email: sanjay.kalra@ualberta.ca 


\section{INTRODUCTION}

Amyotrophic lateral sclerosis (ALS) is a progressive neurological condition with a hallmark pathology of motor neuron degeneration. Patients experience gradual paralysis of the limbs, speech dysfunction, and swallowing difficulties. ${ }^{1}$ The precise mechanisms of motor neuron degeneration are unknown at present; however, $5 \%-10 \%$ of ALS cases are familial, with mutations recognized in SOD $1,{ }^{2}$ TARDBP ${ }^{3} \mathrm{FUS},{ }^{4}$ and $C 9 O R F 72^{5,6}$ genes. In addition to motor cortex ${ }^{7}$ and corticospinal tract $(\mathrm{CST})^{8,9}$ degeneration, frontotemporal lobar degeneration ${ }^{10,11}$ is present in a significant number of patients.

The clinical presentation of ALS involves a combination of upper and lower motor neuron (UMN and LMN) signs and symptoms, and features of cognitive and behavioral impairment in a subset of patients. There is no diagnostic test for ALS, and clinicians rely on the identification of subjective and insensitive signs of UMN and LMN dysfunction for diagnosis. ${ }^{12}$ Electromyography can provide sub-clinical evidence of LMN involvement. Studies have explored the potential of neuroimaging techniques to provide objective evidence of UMN or cerebral involvement. These report global and regional brain atrophy, ${ }^{11,13}$ and hyperintensity of the $\mathrm{CST}^{14,15}$ and hypointensity of the precentral gyrus ${ }^{14,15}$ on T2weighted and fluid-attenuated inversion recovery (FLAIR) images; however, these changes have inadequate accuracy to be reliable indices of cerebral degeneration to confirm diagnosis or monitor progression. Thus, routine MRI only serves to exclude diseases that mimic ALS. ${ }^{16}$ An objective biomarker is needed that can aid in evaluating cerebral degeneration and provide a tool to evaluate novel treatments in clinical trials.

Texture analysis (TA) is a computational image processing method that quantifies pixel interrelationships and patterns ("texture features") that may be indiscernible to the human eye. ${ }^{17}$ Texture features are best understood as properties of an image such as its roughness, smoothness, brightness, and contrast and can be used to classify medical images according to pathology. In a multiple sclerosis study, TA successfully distinguished between patients with active lesions from those with non-active lesions. ${ }^{18}$ In patients with epilepsy, TA demonstrated $83 \%$ sensitivity and $100 \%$ specificity in identifying focal cortical dysplasia. ${ }^{19}$ Texture analysis has also been used in evaluating brain tumors ${ }^{20,21}$ and detecting cerebral changes in Alzheimer's disease. ${ }^{22}$ Recently, using a novel three-dimensional (3D) method, ${ }^{22}$ we demonstrated changes in texture features on T1-weighted images in ALS. ${ }^{23}$ The objective of this study was to determine whether TA could improve upon the ability to classify T2-weighted images. We hypothesized that TA can detect cerebral changes in T2-weighted images with greater accuracy than by visual inspection alone. Our second hypothesis was that the performance of TA is dependent on image resolution, with higher-resolution images yielding better results.

\section{Materials ANd Methods}

\section{Subjects}

The study was approved by the local institutional research ethics board, and all participants provided written consent. We recruited patients with UMN signs on examination who had a diagnosis of possible ALS, probable lab-supported ALS, probable ALS, or definite ALS according to the El Escorial criteria ${ }^{12}$ from a multidisciplinary ALS clinic. None of the subjects had a family history of ALS, or frontotemporal dementia. Subjects were ineligible if they had a history of other neurological disorders or a contraindication for MRI. Finger- and toe-tapping rates, which are clinical measures of UMN function, were calculated by averaging the total number of taps in 10 seconds over two trials. Scores from the left and the right side within patients did not differ significantly and therefore were averaged to give a single score for each patient. Disease severity was assessed using the ALS Functional Rating Scale-Revised (ALSFRS-R), which ranges from 0 to 48 , with lower scores indicating increasing disability. Patients' disease duration was defined as the time between the onset of symptoms and the MRI scan. We also recruited age-matched healthy controls without neurological or psychiatric diseases.

\section{MRI Data Acquisition}

MRI was conducted on a $4.7 \mathrm{~T}$ whole-body scanner (Varian Unity Inova console; Varian, Palo Alto, CA, USA). High-resolution, 2D fast-spin echo T2-weighted images were acquired $(\mathrm{TR}=4000 \mathrm{~ms}, \mathrm{TE}=33.3 \mathrm{~ms}$, echo spacing $=16.7 \mathrm{~ms}, 8$ echoes, voxel size $=0.5 \times 0.5 \mathrm{~mm}^{2}$, slice thickness $=2 \mathrm{~mm}$, no slice gap, matrix size $=385 \times 512$, number of slices $=20$, acquisition time $=4$ minutes) with a coronal angulation that yielded images with the region of the precentral gyrus rostrally and the descending CST through to the cerebral peduncles caudally; this maximized inclusion of disease-relevant regions of the brain.

\section{Image Processing}

Images were down-sampled to $1 \times 1,2 \times 2,3 \times 3$, and $4 \times 4$ $\mathrm{mm}^{2}$ in-plane spatial resolutions using the bicubic interpolation algorithm in ImageJ (http://rsbweb.nih.gov/ij/). Non-uniform image intensity was corrected using bias-field correction in SPM8 (http://www.fil.ion.ucl.ac.uk/spm). The single coronal slice that maximally included the precentral gyrus and the CST, including the internal capsule, was selected for each subject under the supervision of a neurologist; this slice was masked by manually drawing an region of interest (ROI) from the cortex to the peduncles using ITK-SNAP (http://www.itksnap.org). The ROI included gray and white matter (GM and WM) (Figure 1). This anatomical region was selected for TA to (a) include maximally affected structures in ALS and (b) exclude the skull. The mean pixel intensity was calculated from the histogram of the ROI from patients and controls to ensure the absence of image intensity differences that may affect the extracted texture features.

\section{Texture Analysis}

Texture features were calculated from the ROI using in-housedeveloped software for SPM8. Features were estimated using the gray-level co-occurrence matrix (GLCM) technique, which is a second-order statistical method for feature extraction. ${ }^{24}$ Graylevel co-occurrence matrix was selected to estimate texture features because statistical methods of calculating features achieve higher discriminatory results than structural methods in medical imaging. ${ }^{25}$ A GLCM is designed based on the relationships and the frequencies of pairs of pixel gray levels that occur within a neighborhood of pixels in an image. ${ }^{17}$ Pixel intensities within the ROI were scaled down, or quantized, to 16 gray levels to reduce computation time. The GLCM was created for gray levels over a 

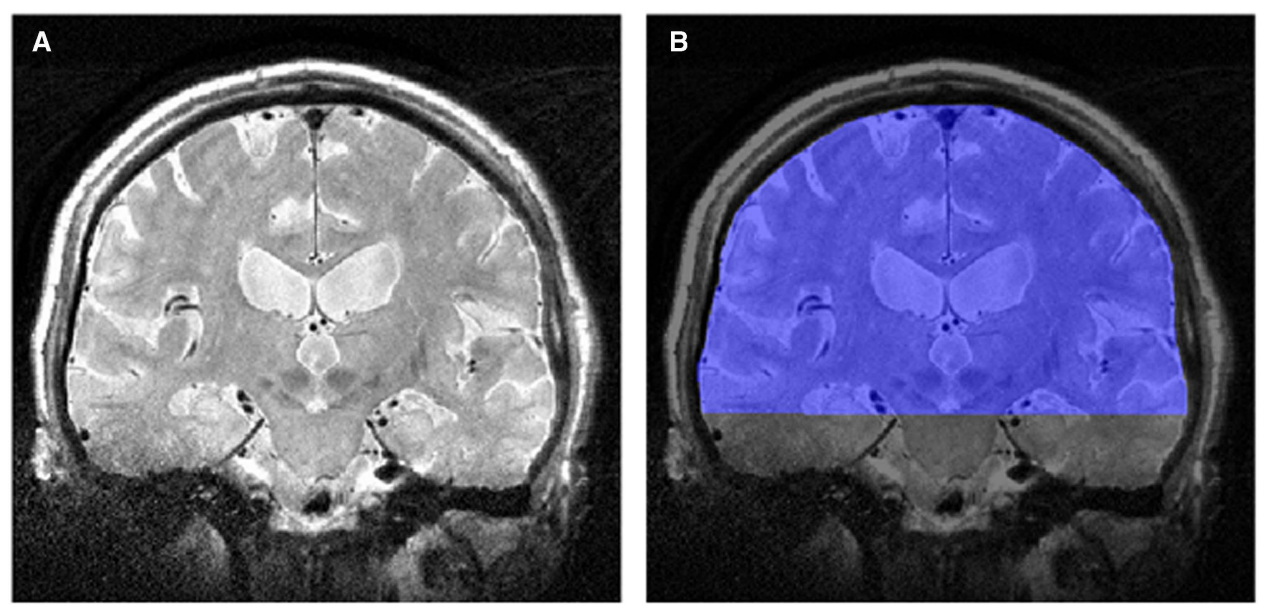

Figure 1: (A) T2-weighted image in the coronal plane, and (B) the region of interest (in blue) for the texture analysis.

pixel distance of 1 and averaged over four directions $\left(0^{\circ}, 45^{\circ}, 90^{\circ}\right.$, and $135^{\circ}$ ). Texture features were calculated and extracted from the GLCM, reflecting various pixel gray-level properties and relationships of the original image. In total, 22 different texture features $\left(f_{1}, f_{2}, \ldots\right.$, and $\left.f_{22}\right)$ were extracted from the GLCM (see Supplemental Table 1).

\section{Visual Review of MRI}

MRI were initially classified independently by two of the authors blind to the subjects' diagnosis into either the patient or control group. These evaluators were not involved with conducting the TA. Subjects were classified as patients if there was, in the opinion of the evaluator, abnormal hyperintensity along the CST. The CST was selected because it is the anatomical region that is frequently assessed on routine clinical MRI studies for suspected ALS patients. Next, both authors convened and agreed upon a final classification of participants. Data sets at the native resolution and the optimal resolution based on the texture features' performance results were used for this analysis.

\section{Statistical Analysis}

The non-parametric Mann-Whitney $U$ test was used to test for differences in texture features between patients and controls. Associations between texture features and clinical measures were examined using Spearman's rank correlation. Type I errors were controlled by using the false discovery rate method for multiple comparison correction and statistical significance was set at an adjusted $p<0.05$ for each statistical test. Uncorrected significance levels of $p<0.05$ are reported where results were not significant after correction for multiple comparisons. Texture features' performance at different image resolutions was investigated by comparing area under the curve (AUC) of their receiver operating characteristic (ROC) curve. Classification results from visual analysis were evaluated using sensitivity and specificity scores along with ROC curve analysis. Logistic regression models were used to investigate the classification performances of (a) combining top-performing texture features and (b) combining those texture features with expert visual classification of subjects. The cutoff value for optimal sensitivity and specificity values for each test was determined

Table 1: Table shows the characteristics of the participants in our study

\begin{tabular}{l|c|c}
\hline & ALS & Controls \\
\hline$N$ & 12 & 19 \\
\hline Age (years [mean \pm SD]) & $57.4 \pm 10.0$ & $57.0 \pm 10.5$ \\
\hline Male:female & $7: 5$ & $8: 11$ \\
\hline Onset (limb/bulbar) & $8 / 4$ & - \\
\hline Disease duration (months [median \pm IQR, range]) & $13.5 \pm 6.8,5-86$ & \\
\hline ALSFRS-R score (mean \pm SD, range) & $37.8 \pm 8.9,14-46$ & - \\
\hline Finger tapping (mean \pm SD, range) & $40.6 \pm 15.9,10.7-60.7$ & - \\
\hline Toe tapping (mean \pm SD, range) & $28.9 \pm 14.6,12.0-53.7$ & - \\
\hline
\end{tabular}

ALS = amyotrophic lateral sclerosis; ALSFRS-R = ALS Functional Rating Scale-Revised; IQR = interquartile range.

Median of the disease duration is presented to give an accurate presentation, as one patient had a disease duration of 86 months at the time of the scan, which skewed the mean disease duration. 
Table 2: Texture features that were significantly different on T2-weighted MRI between amyotrophic lateral sclerosis patients and healthy controls at different resolutions

\begin{tabular}{|c|c|}
\hline $\begin{array}{l}\text { Image resolution } \\
\left(\mathbf{m m}^{2}\right)\end{array}$ & Features \\
\hline $0.5 \times 0.5$ & $\mathrm{~ns}$ \\
\hline $1 \times 1$ & $f_{8}^{*}, f_{9} *, f_{10} *, f_{11} *, f_{12} *, f_{16} *$ \\
\hline $2 \times 2$ & $\frac{f_{2}^{* *}, f_{7}^{* *}, f_{8}^{* *}, f_{9}^{* *}, f_{10} * *, f_{11} * *, f_{12} * *, f_{17} * *, f_{18} * *, f_{21} * *,}{f_{22} * *}$ \\
\hline $3 \times 3$ & $f_{8}^{*}$ \\
\hline $4 \times 4$ & $\mathrm{~ns}$ \\
\hline
\end{tabular}

$\mathrm{ns}=$ not significant

$* p<0.05 ; * *$ false discovery rate-corrected $p<0.05$.

using the Youden index in MedCalc for Windows, version 16.4.3 (MedCalc Software, Ostend, Belgium). All other statistics were carried out using SPSS for Windows, version 22 (IBM Corp. Armonk, NY).

\section{Results}

\section{Demographics}

In total, 12 ALS patients $(57.4 \pm 10.0$ years $)$ and 19 agematched healthy controls $(57.0 \pm 10.5$ years $)$ were recruited. There were seven and eight male ALS and control participants, respectively. The median disease duration was $13.5 \pm 6.8$ months, with a mean ALSFRS-R score of $37.8 \pm 8.9$. Table 1 summarizes the participants' characteristics.

\section{Textural Differences Between ALS Patients and Healthy Controls}

There was no statistically significant difference $(p=0.16)$ in the mean pixel intensities from the histogram of the ROIs between ALS patients $(2676.41 \pm 302.97)$ and healthy controls $(2919.49 \pm$ 531.84). Differences in texture between patients and healthy controls were dependent on image resolution (Table 2). At $2 \times 2 \mathrm{~mm}^{2}$,
11 features were different in ALS after correction for multiple comparisons (Table 3 ). Images at $1 \times 1 \mathrm{~mm}^{2}$ did not have differences in texture features when corrected for multiple comparisons; however, at uncorrected $p<0.05$, seven features were different between ALS patients and healthy controls. Images at $3 \times 3$ and $4 \times 4 \mathrm{~mm}^{2}$ demonstrated no differences in texture; however, at $3 \times 3 \mathrm{~mm}^{2}$ resolution, one feature was different at uncorrected $p<0.05$. Texture features were not different at either corrected or uncorrected significance levels at the $0.5 \times 0.5 \mathrm{~mm}^{2}$ resolution.

\section{Correlations between Clinical Measures and Texture Features}

Correlations were dependent on image resolution (Table 4). Texture features $f_{2}, f_{17}$, and $f_{22}$ demonstrated significant correlations with toe-tapping after correcting for multiple comparisons at $0.5 \times 0.5 \mathrm{~mm}^{2}$ and $1 \times 1 \mathrm{~mm}^{2}$ resolutions. Table 4 shows the correlations between clinical measures and texture features at uncorrected significance.

\section{Classification Performance}

Texture features that were different between ALS patients and healthy controls after correcting for multiple comparisons were selected for ROC analysis to examine their diagnostic accuracy. Features $f_{2}, f_{7}, f_{8}, f_{9}, f_{10}, f_{11}, f_{12}, f_{17}, f_{18}, f_{21}$, and $f_{22}$ showed significant differences at $2 \times 2 \mathrm{~mm}^{2}$ and were selected for further analysis. An AUC was calculated for each feature at all five resolutions.

Every feature improved in its performance (with increasing AUC), with decreasing image resolution from $0.5 \times 0.5 \mathrm{~mm}^{2}$ to $1 \times 1 \mathrm{~mm}^{2}$ to $2 \times 2 \mathrm{~mm}^{2}$ (Figure 2). A peak in the AUCs was observed at $2 \times 2 \mathrm{~mm},{ }^{2}$ after which the features' AUCs declined with further reductions in resolution. The exceptions to this trend were features $f_{8}$ and $f_{12}$ that peaked at $1 \times 1 \mathrm{~mm}^{2}$ and declined with decreasing resolution. The highest AUCs were achieved at $2 \times 2 \mathrm{~mm}^{2}$ by features $f_{10}(0.785)$ and $f_{7}(0.781)$, both with a sensitivity and specificity of $100 \%$ and $58 \%$, respectively. In addition, at $2 \times 2 \mathrm{~mm}^{2}$ AUCs for all features were clustered around the mean AUC, whereas at other resolutions a wider spread of values

Table 3: Significant texture features at $2 \times 2 \mathrm{~mm}^{2}$ resolution

\begin{tabular}{|c|c|c|c|c|c|c|}
\hline Features & ALS & Controls & $p$ value & Sensitivity (\%) & Specificity (\%) & $\mathbf{A U C}$ \\
\hline$f_{2}$ & $4.76 \pm 0.34$ & $4.35 \pm 0.54$ & 0.03 & 83.3 & 73.7 & 0.759 \\
\hline$f_{7}$ & $1.45 \pm 0.07$ & $1.35 \pm 0.10$ & $<0.01$ & 100 & 57.9 & 0.781 \\
\hline$f_{8}$ & $0.03 \pm 0.01$ & $0.04 \pm 0.01$ & $<0.01$ & 83.3 & 73.7 & 0.763 \\
\hline$f_{9}$ & $4.17 \pm 0.13$ & $4.01 \pm 0.17$ & $<0.01$ & 83.3 & 68.4 & 0.759 \\
\hline$f_{10}$ & $0.57 \pm 0.02$ & $0.89 \pm 0.02$ & $<0.01$ & 100 & 57.9 & 0.785 \\
\hline$f_{11}$ & $0.52 \pm 0.02$ & $0.55 \pm 0.02$ & $<0.01$ & 91.7 & 63.2 & 0.772 \\
\hline$f_{12}$ & $0.10 \pm 0.03$ & $0.12 \pm 0.02$ & 0.02 & 83.3 & 68.4 & 0.763 \\
\hline$f_{17}$ & $4.76 \pm 0.34$ & $4.35 \pm 0.54$ & 0.03 & 83.3 & 73.7 & 0.759 \\
\hline$f_{18}$ & $1.61 \pm 0.04$ & $1.56 \pm 0.06$ & 0.02 & 100 & 47.4 & 0.750 \\
\hline$f_{21}$ & $0.92 \pm 3.69 \times 10^{-3}$ & $0.93 \pm 5.00 \times 10^{-3}$ & $<0.01$ & 100 & 52.6 & 0.772 \\
\hline$f_{22}$ & $0.98 \pm 1.18 \times 10^{-3}$ & $0.99 \pm 1.81 \times 10^{-3}$ & 0.02 & 75.0 & 78.9 & 0.763 \\
\hline
\end{tabular}

$\mathrm{ALS}=$ amyotrophic lateral sclerosis; $\mathrm{AUC}=$ area under the curve.

Values are shown as mean \pm SD along with their receiver operator curve analysis results. 
Table 4: Correlations between clinical measures and texture features at different resolutions

\begin{tabular}{|c|c|c|c|}
\hline Image resolution $\left(\mathrm{mm}^{2}\right)$ & ALSFRS-R & Finger-tapping score & Toe-tapping score \\
\hline $0.5 \times 0.5$ & Ns & $\mathrm{ns}$ & $f_{2} * *(0.74), f_{17} * *(0.74), f_{22} * *(0.74)$ \\
\hline $1 \times 1$ & Ns & $f_{9} *(0.61), f_{14} *(0.62), f_{15} *(0.60)$ & $f_{2} * *(0.78), f_{17} * *(0.78), f_{22} * *(0.76)$ \\
\hline $2 \times 2$ & $\begin{array}{c}f_{8} * *(0.61), f_{10} *(0.68), f_{11} *(0.72), \\
f_{21}(0.61)\end{array}$ & $f_{18} *(0.62)$ & ns \\
\hline $3 \times 3$ & Ns & $\begin{array}{c}f_{7} *(0.61), f_{9} *(0.63), f_{10} *(0.59), f_{11} *(0.62), f_{18} *(0.65), f_{21} *(0.59) \\
f_{22} *(0.63)\end{array}$ & $\begin{array}{c}f_{7} *(0.62), f_{10} *(0.68), f_{11} *(0.69) \\
f_{21} *(0.66)\end{array}$ \\
\hline $4 \times 4$ & Ns & ns & ns \\
\hline
\end{tabular}

ALSFRS-R = ALS Functional Rating Scale-Revised; ns = not significant. Spearman's rank correlation coefficient is provided in brackets.

$* p<0.05 ; * *$ false discovery rate-corrected $p<0.05$.

was observed. Average AUCs of the texture features at each resolution are presented in Table 5 .

The $0.5 \times 0.5 \mathrm{~mm}^{2}$ and $2 \times 2 \mathrm{~mm}^{2}$ resolution data sets underwent a visual examination and consensus classification by two experts. Ten of the 31 subjects were classified as patients from the $0.5 \times 0.5 \mathrm{~mm}^{2}$ data set, of whom seven were true positives. The remaining 21 subjects were classified as controls, of whom 16 were true negatives. Sensitivity and specificity were $58 \%$ and $84 \%$, respectively, and AUC was 0.713 . From the $2 \times 2 \mathrm{~mm}^{2}$ data set, nine subjects were classified as patients, of whom five were true positives. In total, 22 subjects were classified as controls, of whom 15 were true negatives. This data set had a sensitivity of $42 \%$, specificity of $79 \%$, and AUC of 0.603 .

Two combination diagnostic models were also assessed for their classification performance. In model 1 , texture features $f_{10}$ and $f_{7}$ at $2 \times 2 \mathrm{~mm}^{2}$ were selected as they had the highest AUCs of 0.785 and 0.781 , respectively, each with a sensitivity of $100 \%$ and specificity of $58 \%$. Combined, these features had a sensitivity and specificity of $92 \%$ and $63 \%$, respectively, with an AUC of 0.785 . In model 2 , texture features $f_{10}$ and $f_{7}$ were combined with human classification of the $2 \times 2 \mathrm{~mm}^{2}$ images, resulting in a higher AUC of 0.851 with sensitivity and specificity at $83 \%$ and $79 \%$, respectively. The Hosmer and Lemeshow test suggested that both models were a good fit to the data $(p>0.05)$.

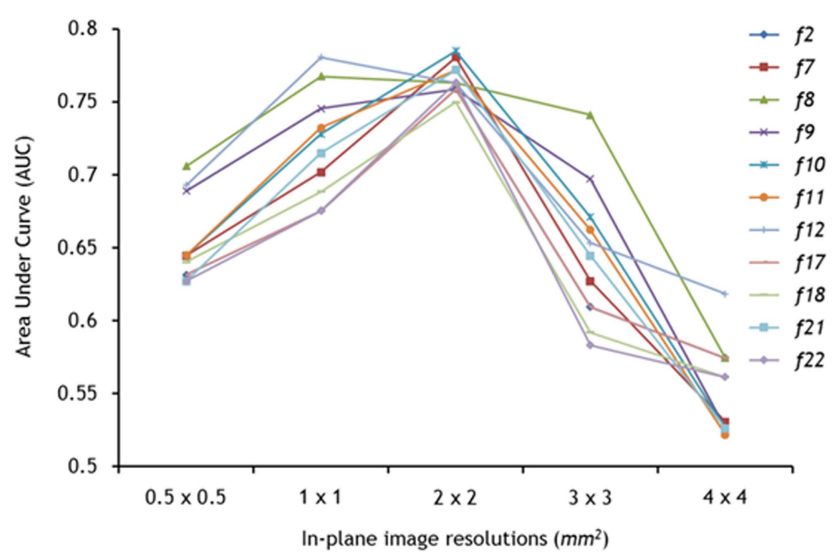

Figure 2: The discriminatory ability of texture analysis was dependent on image resolution. Nine of 11 texture features followed a trend with superior classification (highest area under the curve) peaking at $2 \times 2$ $\mathrm{mm}^{2}$.

\section{DISCUSSION}

In this study, we demonstrated the presence of differences in MRI-based texture features in ALS patients. Texture features also correlated with clinical measures of disability and UMN function. To the best of our knowledge, there have not been other studies in the literature reporting TA on T2-weighted MRI in ALS.

Neuroimaging studies have examined morphological and microstructural alterations in ALS. Region of interest-based and voxel-based analyses of T1-weighted MRI have demonstrated cortical thinning and a reduction in the GM density of the precentral gyrus. ${ }^{7,8}$ Furthermore, WM microstructural alterations characterized by decreased fractional anisotropy and increased mean diffusivity have been noted in the CST and extra-motor regions of the brain with diffusion tensor imaging. ${ }^{8,9}$ These MRI metrics have elucidated the in vivo patterns of cerebral degeneration in ALS and have been proposed as potential biomarkers for the disease. ${ }^{26,27}$ However, these markers and advanced MRI have not yet been validated and are not routinely acquired clinically. Texture analysis can be performed on clinically acquired sequences such as T1- and T2-weighted MRI, and it has been shown to correlate with pathological changes in other diseases. ${ }^{28}$

Prior studies on T2-weighted imaging in ALS report hyperintensities along the CST with poor diagnostic potential. A recent study found the evaluation of hyperintense regions along the CST in ALS to have a diagnostic accuracy of $46.4 \%$, sensitivity of $43.8 \%$, and a specificity of $60.7 \% .^{29}$ In another study, T2weighted images were found to have $62.5 \%$ sensitivity when distinguishing definite and probable ALS patients from controls. $^{30}$ Visual assessment of CST hyperintensity in our data sets by expert reviewers yielded similar results; however, TA

Table 5: Image resolution dependence of the average area under the curve (AUC) of 11 texture features $\left(f_{2}, f_{7}, f_{8}, f_{9}\right.$, $f_{10}, f_{11}, f_{12}, f_{17}, f_{18}, f_{21}$, and $f_{22}$ ) that were significant at $2 \times 2 \mathbf{~ m m}^{2}$ after correction for multiple comparisons

\begin{tabular}{l|c}
\hline Image resolution $\left(\mathbf{m m}^{2}\right)$ & Average AUC \\
\hline $0.5 \times 0.5$ & 0.653 \\
\hline $1 \times 1$ & 0.717 \\
\hline $2 \times 2$ & 0.766 \\
\hline $3 \times 3$ & 0.644 \\
\hline $4 \times 4$ & 0.554 \\
\hline
\end{tabular}


outperformed their classification: $f_{10}$ and $f_{7}$ each had a sensitivity of $100 \%$, although the specificity was $58 \%$. By contrast, visual inspection yielded classification with poor sensitivity and moderate specificity. The greatest classification with an AUC of 0.851 was achieved with the combination of texture features $f_{10}$ and $f_{7}$ and expert classification of images at $2 \times 2 \mathrm{~mm}^{2}$ resolution. This was an improvement over using texture features and expert classification in isolation that yielded AUCs of 0.785 and 0.603 , respectively.

These findings support the potential of TA of MRI images in discriminating ALS patients from healthy controls. These preliminary results suggest that there may be a role for TA to be paired with an expert clinician in the diagnosis of ALS. One approach would be to use a texture feature as a screening measure to first select cases as it has very high sensitivity, followed by confirmation by visual inspection of images by a clinician.

The greatest number of significantly different texture features between ALS patients and controls were present at $2 \times 2 \mathrm{~mm}^{2}$ resolution, which suggests the presence of pronounced T2weighted textural changes at this resolution. In addition, the performance of the texture features, as measured by their AUCs, demonstrated less variability and converged at the $2 \times 2 \mathrm{~mm}^{2}$ resolution. These findings have several important implications. First, high-resolution imaging is time-consuming and not feasible in routine clinical practice; yet, our results show that TA is most robust at the resolution acquired in clinical imaging. Second, a unitary set of texture features could be used in clinical trials as a marker for cerebral changes.

Several studies in the literature have attempted to delineate the effect of imaging parameters on TA. In a phantom MRI study, TA achieved a classification accuracy of over $90 \%$ accuracy even in low-resolution images. ${ }^{31}$ Studies addressing slice thickness suggest that a thin image slice thickness is not required for high TA performance. ${ }^{21,32}$ Together, these studies support our findings, suggesting that optimal TA performance may not require highresolution images.

The pattern of TA performance in our study could be caused by changes in the signal-to-noise rate (SNR) because of changes in image resolution. Post hoc SNR analysis of our data set showed a rise in SNR with decreasing resolution (data not shown). This is expected, as with down-sampling to lower resolutions noise is reduced, but signal remains unchanged. Increasing SNR does not fully explain the peak TA performance at $2 \times 2 \mathrm{~mm}^{2}$, as it declined at higher SNRs (lower resolutions above $2 \times 2 \mathrm{~mm}^{2}$ ). The unique pattern of TA performance across different resolutions is likely a product of a variety of factors in addition to SNR, including TArelevant parameters such as neighborhood size, pixel distance, and quantization levels. We used a pixel distance of 1 , which translates to $0.5 \mathrm{~mm}$ in the $0.5 \times 0.5 \mathrm{~mm}^{2}$ resolution data set and $2 \mathrm{~mm}$ in the $2 \times 2 \mathrm{~mm}^{2}$. This could indicate that signal changes occur on a larger scale in ALS. To investigate this and uncover the robustness of TA, future studies should study effects of changing TA parameters at different image resolutions on classification accuracy in clinical data sets.

Previously we have shown that TA in T1-weighted images showed significant textural changes in the CST and the precentral gyrus using a novel 3D voxel-wise approach. ${ }^{23}$ In the present study, however, we did not acquire whole-brain T2-weighted images, which restricted our analysis to 2D. A study that used TA in ALS evaluated texture features from deep gray nuclei (DGN) structures in T1-weighted $\mathrm{MRI}^{33}$ and found significant changes in texture features $f_{3}, f_{4}$, and $f_{17}$ between patients and controls. In our study, we observed different texture features to be significant because our ROI included the CST and was not specific to DGN structures. Various regions of the brain could have discrete textural changes depending on the imaging modality and local pathology. Very few studies have examined the relationship between MRI measures and pathological findings in ALS. An early study cited astrocytosis and iron deposition as the cause of T2-weighted signal changes in the motor cortex. ${ }^{34}$ Neuronal loss, myelin dysfunction, and astrocytosis have also been reported to cause altered T1-weighted signals in the motor cortex and subcortical WM of post-mortem ALS patients. ${ }^{35}$ As texture features characterize MRI signal alterations, it is possible that the observed changes in texture are caused in part by the pathological correlates observed in previous studies.

There are several limitations in this study. First, we used an ROI that covered regions from the cortex to the cerebral peduncles and included GM and WM, whereas the visual assessment was performed primarily on the CST. Images could not be segmented into GM and WM owing to sub-optimal GM/WM contrast on T2-weighted images. Smaller region-specific ROIs were not compatible with TA at lower resolutions, because the neighborhood size was larger than the ROI. Observations from this study could therefore be from any tissue class in the ROI, and future studies should aim to perform TA on structure-specific locations. Second, recent studies have demonstrated improved sensitivity for detecting signal intensity changes using optimized T2-weighted FLAIR and susceptibility-weighted images. ${ }^{36}$ The performance of TA methods could potentially improve if optimized sequences are used. Third, the study has a small sample size from a heterogeneous disease population. A larger sample with an equal number of patients and gendermatched control groups in a future study would provide more robust evidence. Last, data were down-sampled from high to low resolutions instead of additional data being acquired at lower resolutions.

\section{CONCLuSION}

This study demonstrated that TA using GLCM can discriminate between ALS patients and controls with T2-weighted MRI. Performance was optimal at $2 \times 2 \mathrm{~mm}^{2}$ in-plane resolution. We believe that TA has the potential to be a marker for cerebral changes that occur in ALS. Future studies should apply TA methods to other MRI modalities such as magnetization transfer imaging and FLAIR to investigate its diagnostic performance in images with different contrasts. Larger studies with longitudinal data and disease controls are required for further validation.

\section{ACKNOWLEDGMENTS}

This manuscript was supported by research grants from ALS Association, ALS Society of Canada, and CIHR.

\section{Disclosure}

AI, RM, JS, PS, DM, AHW, SN, and Y-HY do not have anything to disclose. SK has the following disclosures: ALS Association, ALS Society of Canada, Canadian Institutes of Health Research.

\section{Statement of Authorship}

AI designed the study, performed the analysis, and wrote the manuscript; RM designed the software for the study; JS performed 
the analysis; PS acquired the MRI data; DM recruited participants for this study; SK conducted the visual assessment for the MRI data; Y-HY conceptualized and designed the study; and SK conducted the visual assessment and conceptualized and designed the study. All authors reviewed and edited the manuscript.

\section{SUPPLEMENTARY MATERIAL}

To view supplementary material for this article, please visit https://doi.org/10.1017/cjn.2018.267

\section{REFERENCES}

1. Kiernan MC, Vucic S, Cheah BC, et al. Amyotrophic lateral sclerosis. Lancet. 2011;377(9769):942-55.

2. Rosen DR, Siddique T, Patterson D, et al. Mutations in cu/zn superoxide dismutase gene are associated with familial amyotrophic lateral sclerosis. Nature. 1993;362(6415):59-62.

3. Sreedharan J, Blair IP, Tripathi VB, et al. TDP-43 mutations in familial and sporadic amyotrophic lateral sclerosis. Science. 2008;319(5870):1668-72.

4. Vance C, Rogelj B, Hortobagyi T, et al. Mutations in FUS, an RNA processing protein, cause familial amyotrophic lateral sclerosis type 6. Science. 2009;323(5918):1208-11.

5. Renton AE, Majounie E, Waite A, et al. A hexanucleotide repeat expansion in C9ORF72 is the cause of chromosome 9p21-linked ALS-FTD. Neuron. 2011;72(2):257-68.

6. DeJesus-Hernandez M, Mackenzie IR, Boeve BF, et al. Expanded GGGGCC hexanucleotide repeat in noncoding region of C9ORF72 causes chromosome 9p-linked FTD and ALS. Neuron. 2011;72(2):245-56.

7. Roccatagliata L, Bonzano L, Mancardi G, Canepa C, Caponnetto C. Detection of motor cortex thinning and corticospinal tract involvement by quantitative MRI in amyotrophic lateral sclerosis. Amyotroph Lateral Scler. 2009;10(1):47-52.

8. Agosta F, Pagani E, Rocca M, et al. Voxel-based morphometry study of brain volumetry and diffusivity in amyotrophic lateral sclerosis patients with mild disability. Hum Brain Mapp. 2007; 28(12):1430-8.

9. Sage CA, Van Hecke W, Peeters R, et al. Quantitative diffusion tensor imaging in amyotrophic lateral sclerosis: revisited. Hum Brain Mapp. 2009;30(11):3657-75

10. Abrahams S, Goldstein LH, Suckling J, et al. Frontotemporal white matter changes in amyotrophic lateral sclerosis. J Neurol. 2005;252(3):321-31.

11. Mezzapesa DM, Ceccarelli A, Dicuonzo F, et al. Whole-brain and regional brain atrophy in amyotrophic lateral sclerosis. AJNR Am J Neuroradiol. 2007;28(2):255-9.

12. Brooks BR, Miller RG, Swash M, Munsat TL. El escorial revisited: revised criteria for the diagnosis of amyotrophic lateral sclerosis. Amyotroph Lateral Scler. 2000;1(5):293-9.

13. Kassubek J, Unrath A, Huppertz H, et al. Global brain atrophy and corticospinal tract alterations in ALS, as investigated by voxelbased morphometry of 3-D MRI. Amyotroph Lateral Scler. 2005;6(4):213-20.

14. Hecht M, Fellner F, Fellner C, Hilz M, Neundörfer B, Heuss D. Hyperintense and hypointense MRI signals of the precentral gyrus and corticospinal tract in ALS: a follow-up examination including FLAIR images. J Neurol Sci. 2002;199 (1):59-65.

15. Ngai S, Tang YM, Du L, Stuckey S. Hyperintensity of the precentral gyral subcortical white matter and hypointensity of the precentral gyrus on fluid-attenuated inversion recovery: variation with age and implications for the diagnosis of amyotrophic lateral sclerosis. AJNR Am J Neuroradiol. 2007;28 (2):250-4.
16. Filippi M, Agosta F, Abrahams S, et al. EFNS guidelines on the use of neuroimaging in the management of motor neuron diseases. Eur J Neurol. 2010;17(4):526-e20.

17. Kassner A, Thornhill RE. Texture analysis: a review of neurologic MR imaging applications. AJNR Am J Neuroradiol. 2010; 31(5):809-16.

18. Yu O, Mauss Y, Zollner G, Namer I, Chambron J. Distinct patterns of active and non-active plaques using texture analysis on brain NMR images in multiple sclerosis patients: preliminary results. Magn Reson Imaging. 1999;17(9):1261-7.

19. Antel SB, Collins DL, Bernasconi N, et al. Automated detection of focal cortical dysplasia lesions using computational models of their MRI characteristics and texture analysis. Neuroimage. 2003;19(4):1748-59.

20. Mahmoud-Ghoneim D, Toussaint G, Constans J, Jacques D. Three dimensional texture analysis in MRI: a preliminary evaluation in gliomas. Magn Reson Imaging. 2003;21(9):983-7.

21. Herlidou-Meme S, Constans J, Carsin B, et al. MRI texture analysis on texture test objects, normal brain and intracranial tumors. Magn Reson Imaging. 2003;21(9):989-93.

22. Maani R, Yang YH, Kalra S. Voxel-based texture analysis of the brain. PloS One. 2015;10(3):e0117759.

23. Maani R, Yang Y, Emery D, Kalra S. Cerebral degeneration in amyotrophic lateral sclerosis revealed by 3-dimensional texture analysis. Front Neurosci. 2016;10:120.

24. Haralick RM, Shanmugam K, Dinstein IH. Textural features for image classification. IEEE Trans Syst Man Cybern. 1973;6: 610-21.

25. Castellano G, Bonilha L, Li L, Cendes F. Texture analysis of medical images. Clin Radiol. 2004;59(12):1061-9.

26. Muller HP, Turner MR, Grosskreutz J, et al. A large-scale multicentre cerebral diffusion tensor imaging study in amyotrophic lateral sclerosis. J Neurol Neurosurg Psychiatry. 2016;87(6): $570-9$.

27. Walhout R, Westeneng HJ, Verstraete E, et al. Cortical thickness in ALS: towards a marker for upper motor neuron involvement. J Neurol Neurosurg Psychiatry. 2015;86(3):288-94.

28. Zhang Y, Moore G, Laule C, et al. Pathological correlates of magnetic resonance imaging texture heterogeneity in multiple sclerosis. Ann Neurol. 2013;74(1):91-9.

29. Cervo A, Cocozza S, Saccà $F$, et al. The combined use of conventional MRI and MR spectroscopic imaging increases the diagnostic accuracy in amyotrophic lateral sclerosis. Eur J Radiol. 2015;84(1):151-7.

30. Charil A, Corbo M, Filippi M, et al. Structural and metabolic changes in the brain of patients with upper motor neuron disorders: a multiparametric MRI study. Amyotroph Lateral Scler. 2009; 10(5-6):269-79.

31. Jirák D, Dezortová M, Hájek M. Phantoms for texture analysis of MR images. Long-term and multi-center study. Med Phys. 2004;31(3):616-22.

32. Savio SJ, Harrison LC, Luukkaala T, et al. Effect of slice thickness on brain magnetic resonance image texture analysis. Biomed Eng Online. 2010;9(1):60.

33. Albuquerque M, Anjos LG, Maia Tavares de Andrade H, et al. MRI texture analysis reveals deep gray nuclei damage in amyotrophic lateral sclerosis. J Neuroimaging. 2016;26(2):201-6.

34. Oba H, Araki T, Ohtomo K, et al. Amyotrophic lateral sclerosis: T2 shortening in motor cortex at MR imaging. Radiology. 1993; 189(3):843-6

35. Meadowcroft MD, Mutic NJ, Bigler DC, et al. Histological-MRI correlation in the primary motor cortex of patients with amyotrophic lateral sclerosis. J Magnetic Reson Imaging. 2015; 41(3):665-75.

36. Vázquez-Costa JF, Mazón M, Carreres-Polo J, et al. Brain signal intensity changes as biomarkers in amyotrophic lateral sclerosis. Acta Neurol Scand. 2018;137(2):262-71. 